\title{
Nuclear localization of MUC1 extracellular domain in breast, head and neck, and colon cancer
}

\author{
Martin E. Rabassa ${ }^{1}$, Marina T. Isla Larrain ${ }^{1}$, Ezequiel Lacunza ${ }^{1}$, Luciano Cermignani ${ }^{1}$, Cecilio G. Alberdi ${ }^{1}$, \\ Sandra O. Demichelis ${ }^{2}$, Martin C. Abba ${ }^{1}$, Amada Segal-Eiras ${ }^{1}$, Maria Virginia Croce ${ }^{1}$ \\ ${ }^{1}$ Center of Basic and Applied Immunological Research, Faculty of Medical Sciences, National University of La Plata, La Plata - Argentina \\ ${ }^{2}$ Department of Productive Development and Technology, University of Lanús, Lanús - Argentina
}

\begin{abstract}
Background: The glycoprotein MUC1 is overexpressed and underglycosylated in cancer cells. MUC1 is translated as a single polypeptide that undergoes autocleavage into 2 subunits (the extracellular domain and the cytoplasmic tail), and forms a stable heterodimer at the apical membrane of normal epithelial cells. The MUC1 cytoplasmic tail localizes to the cytoplasm of transformed cells and is targeted to the nucleus.

Aims: To study the expression of the MUC1 extracellular subunit in cell nuclei of neoplastic breast, head and neck, and colon samples.

Materials and methods: 330 primary tumor samples were analyzed: 166 invasive breast carcinomas, 127 head and neck tumors, and 47 colon tumors; 10 benign breast disease (BBD) and 40 normal specimens were also included. A standard immunohistochemical method with antigen retrieval was performed. Nuclear fractions from tissue homogenates and breast cancer cell lines (ZR-75, MDA-MB-231, MCF7, and T47D) were obtained and analyzed by Western blotting (WB). The anti-MUC1 extracellular subunit monoclonal antibody HMFG1 was used for immunohistochemistry.

Results: 37/166 breast cancer specimens, 5/127 head and neck cancer specimens, 2/47 colon cancer samples, and $3 / 10$ BBD samples showed immunohistochemical staining at the nuclear level. No nuclear reaction was detected in normal samples. By WB, breast and colon cancer purified nuclear fractions showed reactivity at 200 kDa in 3/30 breast and 3/20 colon cancer samples as well as purified nuclear fractions obtained from breast cancer cell lines.

Conclusions: This study shows that the MUC1 extracellular domain might be translocated to the cell nucleus in breast, head and neck, and colon cancer as well as BBD.
\end{abstract}

Keywords: MUC1 extracellular domain, Neoplasia, Nuclear localization

\section{Introduction}

MUC1 is a large transmembrane glycoprotein expressed by epithelial cells; it is overexpressed and underglycosylated in cancer cells. MUC1 is translated as a single polypeptide that undergoes autocleavage into 2 subunits (the extracellular domain, MUC1-N, and the cytoplasmic tail, MUC1-C) in the endoplasmic reticulum (1). It forms a stable heterodimer at the apical membrane of normal epithelial cells $(2,3)$. The MUC1-N terminal subunit has a protein core mainly com-

Received: June 27, 2014

Accepted: February 18, 2015

Published online: May 13, 2015

Corresponding author:

María Virginia Croce

Center of Basic and Applied Immunological Research

Faculty of Medical Sciences

National University of La Plata

Calle 60 and 120, 1900 La Plata, Argentina

crocevir@hotmail.com posed of a variable number of tandem repeats (VNTR). Each tandem repeat consists of a sequence of 20 amino acids which contains 5 potential glycosylation sites in Ser and Thr $(4,5)$. Normal epithelium presents long carbohydrate chains, while tumor cells display an altered pattern of glycosylation $(6,7)$. With transformation and loss of polarity, MUC1 is found in the cytosol and over the entire plasma membrane of carcinoma cells $(8,9)$. Early research into MUC1 focused on the MUC1-N mucin component and led to development of the CA 15-3 assay to monitor circulating levels of this subunit as a tumor marker (9).

Subsequent research focused on the MUC1-C transmembrane subunit as the potential link between its overexpression and carcinogenesis; it consists of a 58-amino-acid extracellular domain, a 28-amino-acid transmembrane domain, and a 72-amino-acid cytoplasmic tail (10). The MUC1 cytoplasmic tail interacts with a variety of proteins involved in neoplasia and cell adhesion such as the epidermal growth factor receptor (EGFR), erbB2, erbB3, erbB4, c-Src, protein kinase $C$ delta (PKCd), Grb2, $\beta$-catenin, GSK3 $\beta$, and p120ctn (11-17). The MUC1 cytoplasmic domain has proved sufficient to induce transformation; it also localizes to the cytoplasm 
of transformed cells and is targeted to the nucleus and mitochondria $(18,19)$. Furthermore, it was found to be associated with apoptosis resistance in response to oxidative stress (20).

It is frequently assumed that the MUC1 extracellular subunit does not enter the nucleus. In a previous study (21) where we used Western blotting (WB) with the anti-VNTR MUC1 C595 monoclonal antibody (MAb), we detected the MUC1 extracellular domain in nuclear fractions of primary breast cancer samples: a double band at $180 \mathrm{kDa}$ was observed along with a smear reaction from $50-60 \mathrm{kDa}$ up to $200 \mathrm{kDa}$. Based on these observations, we performed the present study with the aim to detect extracellular MUC1 in the cell nuclei of neoplastic tissues.

\section{Materials and methods}

\section{Tissue specimens}

A total of 330 primary tumor samples were analyzed: 166 invasive breast carcinomas, 127 head and neck squamous cell carcinomas (HNSCC), and 47 colon adenocarcinomas. Forty normal tissues were included as controls: 20 mammary samples, 10 colon mucosa specimens, and 10 head and neck mucosa specimens. Ten benign breast disease (BBD) samples were also studied: 5 fibroadenoma (FA) specimens, 3 atypical hyperplasia (ADH) specimens, and 2 nonproliferative disease (NPF) specimens.

Each tissue was studied using routine procedures. Specimens were fixed for histopathological diagnosis and immunohistochemistry (IHC) analysis. Before formalin fixation, a fraction of the tumor sample was rinsed with sterile Hank's balanced solution and processed for preparation of subcellular fractions for SDS-PAGE and WB analysis.

The procedures followed were in accordance with the Helsinki Declaration. Informed consent was obtained from all patients included in this study. The study was approved by the Ethics Approving Committee, Faculty of Medical Sciences, National University of La Plata.

\section{Cell lines}

The breast cancer cell lines T47D, MDA-MB-231, MCF7, and ZR-75 were grown in RPMI 1640 medium (Sigma, USA) supplemented with $10 \%$ fetal calf serum (PAA, Austria) and 100 units/ $\mathrm{mL}$ penicillin and $100 \mu \mathrm{g} / \mathrm{mL}$ streptomycin (Sigma, USA) at $37^{\circ} \mathrm{C}$ in a humidified atmosphere of $95 \%$ air and $5 \% \mathrm{CO}_{2}$.

\section{Antibody}

We used the monoclonal antibody HMFG1; it was developed against the epithelial component of the human milk fat globule membrane, which reacts with the PDTR epitope present in the VNTR of MUC1-N $(22,23)$.

\section{Methods}

\section{Immunohistochemical analysis}

The technique was performed following standard procedures, with modifications (21). Briefly, 4- $\mu$ m-thick sections were deparaffinized and rehydrated; antigen retrieval was performed in $10 \mathrm{mM}$ sodium citrate buffer at $100^{\circ} \mathrm{C}$ for 5 minutes with incubation overnight at $4^{\circ} \mathrm{C}$ with MAb HMFG1, which was employed undiluted. After 3 washes with phosphate buffer, peroxidase-conjugated goat antimouse IgG (Dako, USA) diluted 1:400 was added and incubated for 60 minutes. The slides were counterstained with hematoxylin and coverslipped with mounting medium.

Negative controls were incubated with phosphate-buffered saline (PBS) instead of MAb. Staining of the cytoplasm, plasma membrane, and nucleus was evaluated. Sections were examined by light microscopy and the antibody staining patterns were scored in a semiquantitative manner. The staining intensity was graded as negative $(-)$, low $(+)$, moderate $(++)$, or strong $(+++)(21)$.

The number of low-power $(\times 10)$ optical fields in a specimen that were positively stained was expressed as a percentage of the total number of optical fields containing tissue.

\section{Preparation of subcellular fractions}

Fractions were prepared from breast, colon, and head and neck tumors according to Price et al (23) and Boisvert et al (24). Samples were exhaustively dissected, necrotic areas were discarded, and tumors were cut into $1-\mathrm{mm}$ pieces and extensively washed with $10 \mathrm{mM}$ Tris, pH 7.2. Immediately after, mechanical homogenization was performed with a Polytron (Kinematica, Switzerland) in an ice bath employing the same buffer with $0.01 \mathrm{M}$ protease inhibitor cocktail (Sigma, USA). Homogenates were filtered through a metallic mesh and centrifuged at $600 \times \mathrm{g}$ at $4^{\circ} \mathrm{C}$ for 15 minutes, obtaining a supernatant which corresponds to the cytoplasmic and extranuclear membranes, and a pellet which corresponds to the nuclear subcellular fraction (21). This pellet was resuspended in $0.75 \mathrm{~mL}$ of $0.88 \mathrm{M}$ sucrose, $0.5 \mathrm{mM} \mathrm{MgCl}$ followed by centrifugation at $2800 \times \mathrm{g}$ for 10 minutes at $4^{\circ} \mathrm{C}$. The final pellets (purified nuclear fractions) were resuspended in PBS and the protein content was measured by means of the Bradford method. Finally, fractions were aliquoted and stored at $-70^{\circ} \mathrm{C}$.

\section{Subcellular fractionation of cell lines}

Cells were grown in 10-cm plastic dishes until subconfluence, harvested by trypsinization and then washed 3 times with cold PBS at $300 \times \mathrm{g}$ for 5 minutes at $4^{\circ} \mathrm{C}$. The cells were subsequently incubated at $4^{\circ} \mathrm{C}$ with $2 \mathrm{~mL}$ of $10 \mathrm{mM}$ HEPES pH 7.9 (Sigma, USA), $10 \mathrm{mM} \mathrm{KCl}$ (Merck, Germany), $1.5 \mathrm{mM}$ $\mathrm{MgCl}_{2}$ (Merck, Germany), and $0.5 \mathrm{mM}$ DTT (Sigma, USA) supplemented with a protease inhibitor cocktail (Sigma, USA). Cells were transferred to a dounce homogenizer (Sigma, USA) and 10 strokes were applied while cell lysis was verified under a phase contrast microscope. Homogenized cells were centrifuged at $300 \times \mathrm{g}$ for 5 minutes at $4^{\circ} \mathrm{C}$; thus we obtained a supernatant (cytoplasmic and extranuclear membrane fraction) and a pellet (nuclear fraction) which was applied to a sucrose cushion to obtain the purified nuclear fraction.

Final pellets (purified nuclear fractions) from tumors as well as cell lines were dialyzed against $1.41 \mathrm{M} \mathrm{PBS}$ at $4^{\circ} \mathrm{C}$ for 48 hours, lyophilized, and stored at $-70^{\circ} \mathrm{C}$. Samples were analyzed by SDS-PAGE and WB. 

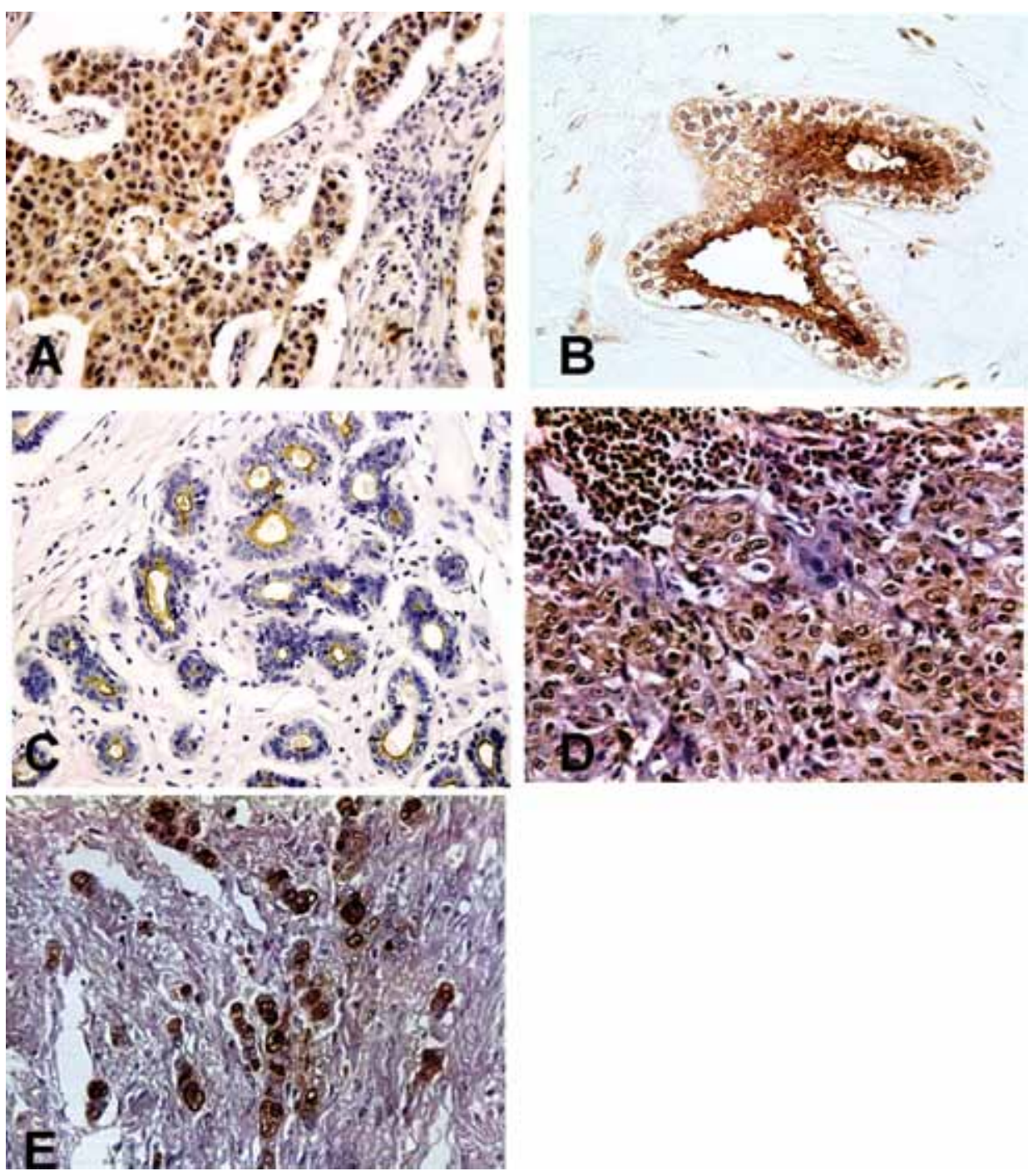

Fig. 1 - A) Positive staining of an invasive ductal carcinoma for MAb HMFG1. A strong nuclear and cytoplasmic reaction is depicted. B) Duct with intact lumen belonging to an fibroadenoma sample incubated with MAb HMFG1; a strong reaction involves the nucleus, apical plasma membrane, and apical cytoplasm of epithelial cells. C) Normal mammary sample incubated with MAb HMFG1; reaction is found in the apical plasma membrane. D) Larynx carcinoma section incubated with MAb HMFG1; most neoplastic cells show strong reactivity at the nuclear and cytoplasmic level. E) Intense positive nuclear staining for MUC1-N of an undifferentiated colon carcinoma detected with MAb HMFG1.

\section{SDS-PAGE and western blotting}

The isolated fractions were analyzed under reducing conditions in SDS-PAGE in a discontinuous buffer system (4\% for the stacking gel and 7\% for the resolving gel). Fractions were suspended in sample buffer and heated at $100^{\circ} \mathrm{C}$ for $5 \mathrm{~min}$ utes; on each lane the protein content loaded was $40 \mu \mathrm{g} / 25$ $\mu \mathrm{L}$. After electrophoresis, gels were electrophoretically transferred onto nitrocellulose membranes, which were blocked with $\mathrm{PBS} / 5 \%$ skimmed milk (blocking buffer), washed and incubated with undiluted MAb HMFG1. After overnight incubation at $4^{\circ} \mathrm{C}$, the blots were rinsed 6 times for 5 minutes in PBS. Then peroxidase-conjugated goat antimouse IgG (Dako, USA) diluted 1:2000 was added. Nitrocellulose sheets were developed employing enhanced chemiluminescence.

\section{Results}

Thirty-seven of 166 (22\%) breast cancer specimens reacted at the nuclear level. The tumor stage distribution was $12 / 37$ (32\%) stage I, $15 / 37$ (40.5\%) stage II, and 10/37 (27\%) stage III. Estrogen and progesterone receptor expression was $84 \%$ and $81 \%$, respectively, whereas HER2/neu expression was found in $9 / 35$ samples (26\%).

Despite nuclear reactivity, $100 \%$ of breast cancer specimens reacted with the anti-MUC1-N MAb HMFG1; most samples showed nonapical, cytoplasmic MUC1 staining with a strong intensity, while several samples showed an apical pattern with a linear, cytoplasmic or mixed reaction. Figure $1 \mathrm{~A}$ shows the HMFG1 reactivity in a breast carcinoma specimen; the nuclei exhibit strong reactivity as does the cytoplasm of most cells.

We also found nuclear reactivity in 3 of 10 BBD samples including $1 \mathrm{FA}, 1 \mathrm{ADH}$ and $1 \mathrm{NPF}$. Figure $1 \mathrm{~B}$ shows a positive nuclear reaction in the FA specimen. In most BBD specimens, HMFG1 stained the apical part of the cytoplasm and plasma membranes, while in normal samples only apical reactivity was detected with no nuclear staining (Fig. 1C).

Forty percent of HNSCC and $95 \%$ of colorectal cancer specimens reacted positively with HMFG1. In most HNSCC and colorectal cancer samples, MUC1 reactivity was mainly restricted to the cytoplasm and the plasma membranes 


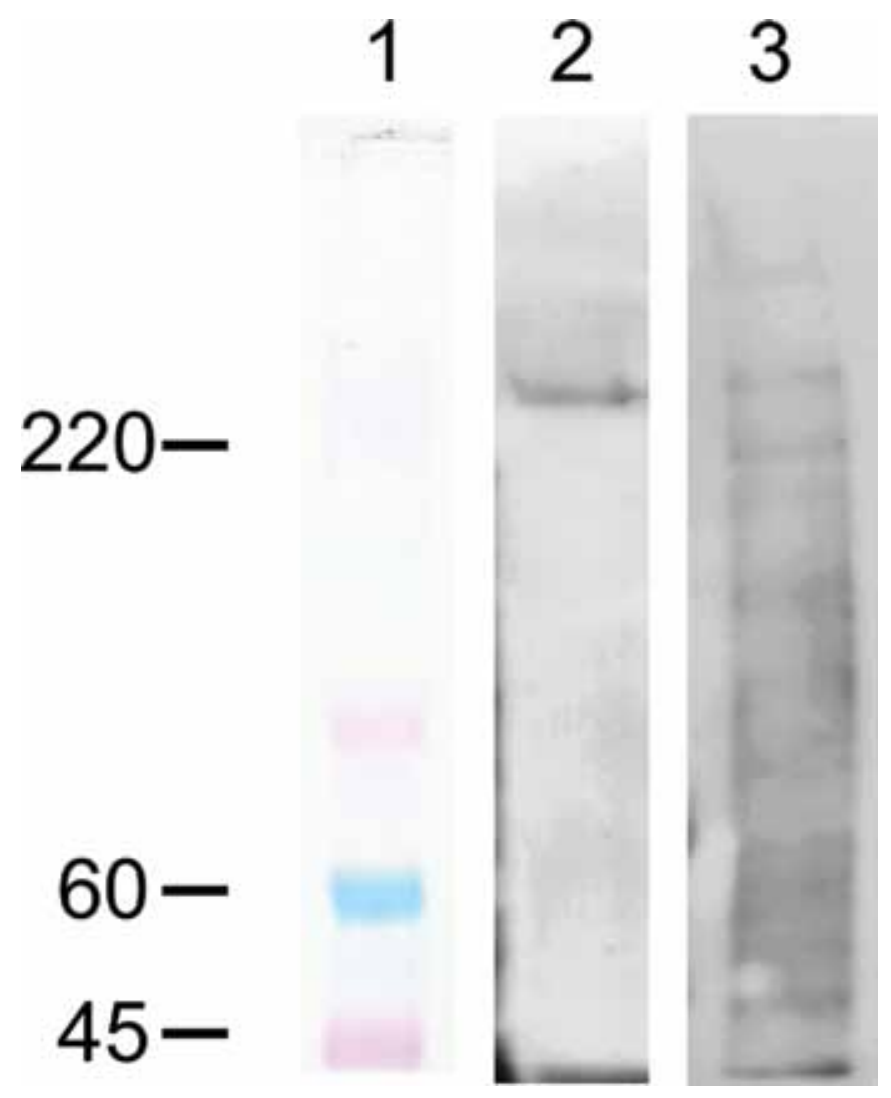

Fig. 2 - Western blot of purified nuclear fractions obtained from a breast cancer (lane 2) and a colon cancer (lane 3) incubated with MAb HMFG1. A >220 kDa band is detected for both samples; the colon cancer sample also shows several low-molecular-weight bands. In lane 1 the molecular weight standard is depicted.

were negative; the intensity varied from moderate to strong. Also, 5/127 (4\%) HNSCC specimens and 2/47 (4\%) colorectal cancer samples showed a nuclear HMFG1-positive reaction. In Figure $1 \mathrm{D}$ and $1 \mathrm{E}$ the reactivity is shown. HMFG1-positive HNSCC tumors were located in the larynx $(4 / 5)$ and pharynx (1/5). Patients with laryngeal tumors were at stage II (1/4) or stage III (3/4) with moderate (1/4) and poor (3/4) histological grade. The patient with pharynx cancer presented at stage IV with a well-differentiated tumor, while patients with colorectal adenocarcinomas were in stages II and III.

Confirmation of nuclear localization was obtained by analyzing nuclear fractions by WB with HMFG1. In 3 of 30 breast cancer samples and 3 of 20 colorectal cancer samples, typical MUC1 extracellular domain reactivity at about 200 kDa was detected. Figure 2 shows a representative experiment of a WB reaction on purified nuclear fractions obtained from breast cancer (lane 2) and colon cancer (lane 3) samples.

Figure $3 A$ shows $W B$ results obtained in 4 breast cancer cell lines (T47D, MDA 231, ZR75, and MCF7). The subcellular fractions assessed included cytoplasmic and extranuclear membrane fractions (lanes 2-5) as well as purified nuclear fractions (lanes 7-10). The reaction with anti-MUC1-N shows the typical bands at about $200 \mathrm{kDa}$. Figure 3B shows the re-

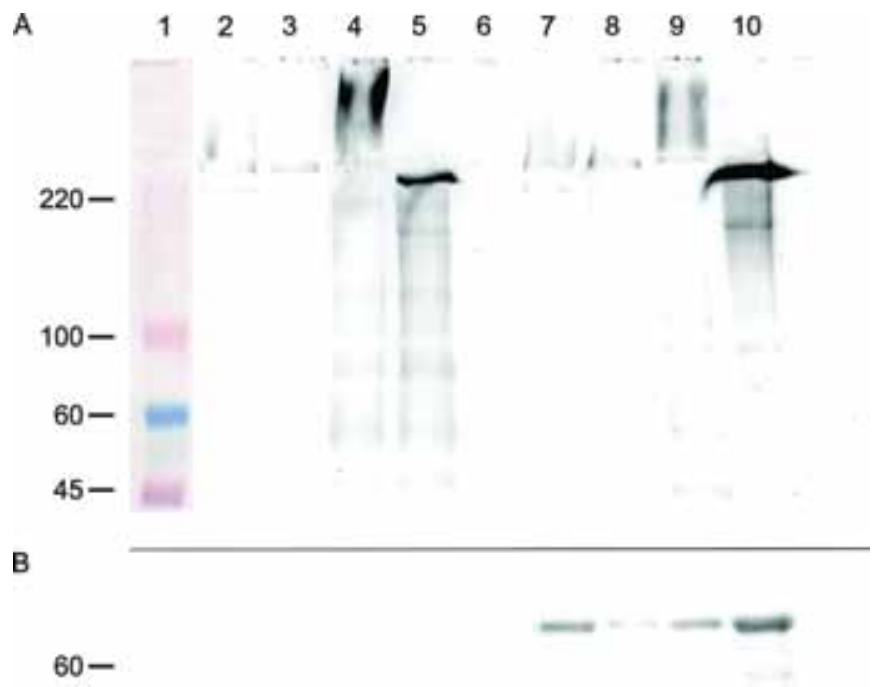

Fig. 3 - Western blot of subcellular fractions belonging to breast cancer cell lines (T47D, MDA-MB-231, MCF7, and ZR-75). A) Incubation with MAb HMFG1. Lane 1 shows the molecular weight standard; lanes 2-5 correspond to supernatants of nuclear pellets (cytoplasmic and membrane fraction), while lanes 7 to 10 show purified nuclear pellets. In lane 6 no sample was run to clearly separate the specimens. In lanes 2-4 and 7-10 the characteristic MUC1 bands at approximately $220 \mathrm{kDa}$ are shown. Other bands as well as smear reactivity are also shown in lanes 2, 4, 5, 7, 9, and 10. B) Incubation with an antibody against lamin (nuclear marker); purified nuclear fractions (lanes 7-10) show a reaction at $>60 \mathrm{kDa}$.

activity of the purified nuclear fractions (lanes 7-10) with a nuclear marker (lamin A).

Eleven nuclear fractions derived from HNSCC as well as those belonging to 2 normal breast tissue samples were not reactive (data not shown).

\section{Discussion}

In previous studies we have studied MUC1 expression in breast $(8,21,25,26)$, head and neck $(27,28)$, and colon cancer samples $(29,30)$, and we found that some specimens showed nuclear reactivity with anti-MUC1-N MAbs. In the present study, using IHC and WB, we found that the anti-MUC1 extracellular domain MAb HMFG1 reacts at the nuclear level in these 3 tumor types, while BBD samples were also reactive. Some samples that showed nuclear positivity with IHC also showed reactivity when WB analysis was used; this indicates that the 2 techniques both provide information although they are not interchangeable.

In line with other reports $(28,31-33)$, when performing SDS-PAGE and WB analysis of subcellular fractions we obtained in some samples typical bands of about $200 \mathrm{kDa}$ and several bands of lower molecular weight as well as smear reactivity at the stacking gel. As Lo-Guidice et al (31) pointed out, these smear reactions may be due to the presence of MUC1 with different grades of glycosylation, while the bands of low molecular weight could correspond to protein degradation.

Many immunohistochemical studies have been performed on normal epithelial and tumor tissues using well-character- 
ized antibodies against the MUC1-N domain, which recognize epitopes within its VNTR region; although antibody reactivity often varies depending on the degree of $O$-linked glycosylation, MUC1 overexpression has been largely described in tumor samples $(9,34,35)$. On normal epithelia, MUC1 VNTR is extensively $O$-glycosylated with long branched glycans, whereas on tumor cells it is markedly underglycosylated with simpler and shorter glycan chains affecting many functions of MUC1 $(6,7)$. The pattern of expression differs between normal and neoplastic cells, being restricted to the apical part of normal cells while tumor samples show a mixed, nonapical reaction $(2,9,36,37)$.

In the case of invasive breast cancer samples, we have found different percentages of reactivity depending on the MAb employed; HMFG1 was the most reactive MAb since almost $100 \%$ of specimens stained, although the MAbs HMFG2 and $\mathrm{C} 595$ also had high reactivity while $\mathrm{SM} 3$ was less reactive $(8,21,25,26)$. In colorectal cancer we found high expression of MUC1-N with C595 and HMFG1 $(25,29,30)$, while the HNSCC MUC1-N expression percentages detected with these MAbs were lower $(27,28)$. These results are confirmed in the series considered in the present report, although the percentage of positivity varied, being $100 \%$ in breast cancer, $94 \%$ in colorectal cancer, and $40 \%$ in HNSCC.

Different mechanisms may be involved in tumor MUC1 overexpression, such as regulatory transcriptional events, epigenetic control (38), and gene amplification (39). Furthermore, the kinetics of membrane trafficking either in the de novo pathway or the endosomal recycling pathway may be altered: endocytosis and intracellular accumulation of fulllength MUC1 without its degradation or cleavage in tumor cells have been reported $(40,41)$.

In general, immunohistochemical and immunocytochemical studies using anti-MUC1-N MAbs failed to detect any nuclear localization. Kumar et al (42) considered that this failure may be due to low magnification images, which preclude adequate assessment of nuclear staining, and also to lack of mention or discussion by the authors; however, in some cases nuclear staining is evident from photomicrographs such as in our own reports in breast cancer $(8,21,37,43)$, BBD (44), colon cancer (29), and larynx carcinoma (27) employing antiMUC1 extracellular domain MAbs. On the other hand, in a study on the importance of MUC1 in the intestinal mucosal barrier to infection, McAuley et al (45) reported translocation of the MUC1 extracellular domain to the nucleus in Campylobacter jejuni-treated HeLa cells as an unexpected finding, and considered a possible role for p53 in MUC1 stabilization and recycling during stress.

Thirkill et al (46) observed MUC1-N MAb B27-29 nucleusassociated staining in normal macaque trophoblast cultures, while progesterone treatment of endometrial cancer cell lines changed their MUC1-N profile with nuclear reactivity (47). Kumar et al (42) consistently found nuclear MUC1-N in all cell types and tissues studied, which comprised the MCF7 cell line, 2 human trophoblast-derived choriocarcinoma cell lines (BeWo and Jar), the COS7.MUC1 cell line, primary cell cultures of macaque trophoblast and normal mammary epithelia (240LB, passage 5-6), and macaque colon sections. They reported intense MUC1-N nuclear staining in the MCF7 and Jar tumor cell lines; this nuclear expression was hetero- geneous for MCF7 and primary cultures of normal human mammary epithelial cells. Employing another anti-MUC1-N antibody, DF3, they found weaker nuclear staining in MCF7 and BeWo cells and no nuclear staining in trophoblasts. Their findings demonstrated that MUC1-N was located in nuclear speckles (interchromatin granule clusters) and closely associated with the spliceosome protein U2AF65. Their results suggested that MUC1-N and MUC1-C have dissimilar intranuclear distribution patterns.

By chromatin immunoprecipitation and real-time PCR, Cascio et al (48) found that MUC1/22TR upregulated IL-6 and TNF- $\alpha$ expression by binding to their promoter regions in an NF-KB p65-dependent manner in both MUC1-transfected cells and human breast cancer cells that express endogenous MUC1. They proposed that tumor-specific short $\mathrm{O}$-glycans or no glycans on the tandem repeats of the VNTR region reduce the size of the molecule and its 3-dimensional configuration, allowing its binding to transcriptional coactivators such as p65 and trafficking to the nucleus, although the specific MUC1 sequence that interacts with p65 was not identified. These authors considered that their findings are not expected to occur in normal epithelial cells, where MUC1 is strictly polarized to the luminal surface and long, branched oligosaccharides conceal the VNTR protein and increase the size of the molecule.

MUC1 protein sequence (NP_001191215.1) analysis using the cNLS Mapper resource (http://nls-mapper.iab.keio.ac.jp) $(49,50)$ identifies 2 predicted bipartite nuclear localization signals (NLSs), one located at the extracellular domain (MUC1-N: APKPATVVTGSGHASSTPGGEKETSATQRS) and the other located at the cytoplasmic tail (MUC1-C: RRKNYGQLDIFPARDTYHPMSEYPTYHTH). Among the MUC1 transcript isoforms described in the NCBI GenBank, 14 present NLSs both in the extracellular domain and cytoplasmic tail while the other isoforms only present NLSs at MUC1-C. In this sense, the MUC1-N NLSs are only encoded by the MUC1 isoforms with a full-length exon 2 sequence, while the MUC1 isoforms without MUC-N NLSs are characterized by a shorter exon 2 mRNA sequence. Interestingly, $\mathrm{Ng}$ et al (51) have reported a single-nucleotide polymorphism at the $5^{\prime}$ end of exon 2 (rs4072037) implicated in the control of the alternative splicing event of this exon sequence; it can lead to a 9-amino-acid insertion affecting the signal peptide region of both full-length MUC1 transcripts and those lacking the tandem repeat domain $(51,52)$. Such evidence raises the possibility that alternative splicing events involving the $5^{\prime}$ end of the exon 2 region could drive the translocation of the MUC1 extracellular domain to the nucleus by inclusion of an NLS.

The longer transcript was reported to be more abundant in cancer tissues (52-55) with variations of the different splice forms according to tumor location, although the overrepresentation of these transcripts may be affected by inflammation as well (51). These variations may also explain the differential MUC1-N detection at the tumor locations considered in our study: breast, colon, and head and neck.

Investigating the possible functions of nuclear MUC1-N localization, Kumar et al (42) found the MUC1 extracellular domain in nuclear speckles and proposed that the dependence of this localization on RNA suggests a possible role in the assembly and/or organization of these structures. 
The present study was performed in human neoplastic tissues. It demonstrates that the MUC1 extracellular domain subunit is reactive at the nuclear level in primary breast, $\mathrm{HN}$ SCC, and colon tumors as well as BBD. BBD reactivity comprised entities with and without an increased risk of breast cancer development. We did not find reactivity in normal tissue samples.

The MUC1 extracellular domain protein core may be involved in transcriptional and posttranscriptional processes associated with stress conditions, although it should not be underestimated that it may account for normal phenomena as well. Thus, the complexity of the MUC1 molecule highlights new biological events and may mediate different functions depending on the context.

\section{Abbreviation}

$\begin{array}{ll}\text { ADH } & \text { atypical hyperplasia } \\ \text { BBD } & \text { benign breast disease } \\ \text { EGFR } & \begin{array}{l}\text { epidermal growth factor receptor } \\ \text { FA }\end{array} \\ \text { fibroadenoma } \\ \text { HNSCC } & \text { head and neck squamous cell carcinoma } \\ \text { IHC } & \text { immunohistochemistry } \\ \text { MAb } & \text { monoclonal antibody } \\ \text { MUC1-C } & \text { MUC1 cytoplasmic tail } \\ \text { MUC1-N } & \text { MUC1 extracellular domain } \\ \text { NLS } & \text { nuclear localization signal } \\ \text { NPF } & \text { nonproliferative disease } \\ \text { PBS } & \text { phosphate-buffered saline } \\ \text { VNTR } & \text { variable number of tandem repeats } \\ \text { WB } & \text { Western blotting }\end{array}$

\section{Acknowledgments}

We gratefully acknowledge the MAb HMFG1 kindly provided by Prof. Joyce Taylor Papadimitriou and Prof. Joy Burchell (King's College, London, UK) and anti-lamin A provided by Prof. Ana Ves Losada (INIBIOLP, La Plata, Argentina).

We thank Dr. W. Servi, Dr. A. Barbera, Dr. F. Terrier, Dr. A. Cretón and Dr. A. Pereyra for their kind collaboration with patients' samples, and Miss María Emilia Cermignani for English correction of the manuscript.

\section{Disclosures}

Financial support: This study was supported by the National University of La Plata, Argentina.

Conflict of interest: The authors declare that they have no financial relationship that may lead to a conflict of interest in relation to the submitted manuscript. Prof. María Virginia Croce, Dr. Marina Isla Larrain and Dr. Martín E. Rabassa are members of the research career of the Comisión de Investigaciones Científicas de la Provincia de Buenos Aires (CIC/PBA).

\section{References}

1. Ligtenberg MJ, Kruijshaar L, Buijs F, van Meijer M, Litvinov SV, Hilkens J. Cell-associated episialin is a complex containing two proteins derived from a common precursor. J Biol Chem. 1992;267(9):6171-6177.

2. Kufe $D$, Inghirami $G$, Abe $M$, Hayes $D$, Justi-Wheeler $H$, Schlom J. Differential reactivity of a novel monoclonal antibody (DF3) with human malignant versus benign breast tumors. Hybridoma. 1984;3(3):223-232.

3. Burchell J, Durbin H, Taylor-Papadimitriou J. Complexity of expression of antigenic determinants, recognized by monoclonal antibodies HMFG-1 and HMFG-2, in normal and malignant human mammary epithelial cells. J Immunol. 1983;131(1):508-513.

4. Gendler S, Taylor-Papadimitriou J, Duhig T, Rothbard J, Burchell J. A highly immunogenic region of a human polymorphic epithelial mucin expressed by carcinomas is made up of tandem repeats. J Biol Chem. 1988;263(26):12820-12823.

5. Siddiqui J, Abe M, Hayes D, Shani E, Yunis E, Kufe D. Isolation and sequencing of a cDNA coding for the human DF3 breast carcinoma-associated antigen. Proc Natl Acad Sci USA. 1988;85(7):2320-2323.

6. Hanisch FG, Peter-Katalinic J, Egge H, Dabrowski U, Uhlenbruck $\mathrm{G}$. Structures of acidic O-linked polylactosaminoglycans on human skim milk mucins. Glycoconj J. 1990;7(6):525-543.

7. Lloyd K, Burchell J, Kudryashov V, Yin BW, Taylor-Papadimitriou J. Comparison of O-linked carbohydrate chains in MUC-1 mucin from normal breast epithelial cells lines and breast carcinoma cell lines. J Biol Chem. 1996;271:33325-33334.

8. Croce MV, Colussi AG, Price MR, Segal-Eiras A. Expression of tumour associated antigens in normal, benign and malignant human mammary epithelial tissue: a comparative immunohistochemical study. Anticancer Res. 1997;17(6D):4287-4292.

9. Hayes DF, Zurawski VR Jr, Kufe DW. Comparison of circulating CA15-3 and carcinoembryonic antigen levels in patients with breast cancer. J Clin Oncol. 1986;4(10):1542-1550.

10. Merlo GR, Siddiqui J, Cropp CS, et al. Frequent alteration of the DF3 tumor-associated antigen gene in primary human breast carcinomas. Cancer Res. 1989;49(24 Pt 1):6966-6971.

11. Pandey P, Kharbanda S, Kufe D. Association of the DF3/MUC1 breast cancer antigen with Grb2 and the Sos/Ras exchange protein. Cancer Res. 1995;55(18):4000-4003.

12. Yamamoto M, Bharti A, Li Y, Kufe D. Interaction of the DF3/ MUC1 breast carcinoma-associated antigen and beta-catenin in cell adhesion. J Biol Chem. 1997;272(19):12492-12494.

13. Li Y, Kufe D. The human DF3/MUC1 carcinoma-associated antigen signals nuclear localization of the catenin p120(ctn). Biochem Biophys Res Commun. 2001;281(2):440-443.

14. Li Y, Kuwahara H, Ren J, Wen G, Kufe D. The c-Src tyrosine kinase regulates signaling of the human DF3/MUC1 carcinomaassociated antigen with GSK3 beta and beta-catenin. J Biol Chem. 2001;276(9):6061-6064.

15. Schroeder JA, Thompson MC, Gardner MM, Gendler SJ. Transgenic MUC1 interacts with epidermal growth factor receptor and correlates with mitogen-activated protein kinase activation in the mouse mammary gland. J Biol Chem. 2001;276(16): 13057-13064.

16. Schroeder JA, Adriance MC, Thompson MC, Camenisch TD, Gendler SJ. MUC1 alters beta-catenin-dependent tumor formation and promotes cellular invasion. Oncogene. 2003;22(9): 1324-1332.

17. Ren J, Li Y, Kufe D. Protein kinase $C$ delta regulates function of the DF3/MUC1 carcinoma antigen in beta-catenin signaling. $J$ Biol Chem. 2002;277(20):17616-17622.

18. Li Y, Yu W-H, Ren J, et al. Heregulin targets $\gamma$-catenin to the nucleolus by a mechanism dependent on the DF3/MUC1 oncoprotein. Mol Cancer Res. 2003;1(10):765-775.

19. Ren J, Agata N, Chen D, et al. Human MUC1 carcinoma-associated protein confers resistance to genotoxic anticancer agents. Cancer Cell. 2004;5(2):163-175.

20. Yin L, Li Y, Ren J, Kuwahara H, Kufe D. Human MUC1 carcinoma antigen regulates intracellular oxidant levels and the apoptotic response to oxidative stress. J Biol Chem. 2003;278(37):3545835464. 
21. Croce MV, Isla-Larrain MT, Rua CE, Rabassa ME, Gendler SJ, Segal-Eiras A. Patterns of MUC1 tissue expression defined by an anti-MUC1 cytoplasmic tail monoclonal antibody in breast cancer. J Histochem Cytochem. 2003;51(6):781-788.

22. Taylor-Papadimitriou J, Peterson JA, Arklie J, Burchell J, Ceriani RL, Bodmer WF. Monoclonal antibodies to epithelium-specific components of the human milk fat globule membrane: production and reaction with cells in culture. Int J Cancer. 1981;28(1):17-21.

23. Price MR, Edwards S, Owainati A, et al. Multiple epitopes on a human breast-carcinoma-associated antigen. Int J Cancer. 1985;36(5):567-574.

24. Boisvert FM, Lamond Al. p53-Dependent subcellular proteome localization following DNA damage. Proteomics. 2010;10(22): 4087-4097.

25. Croce $M$, Price $M$, Segaleiras $A$. Antigen heterogeneity in advanced colorectal and breast cancer. Int J Oncol. 1996;9(3): 459-463.

26. Demichelis SO, Isla-Larrain MT, Cermignani L, Alberdi CG, Segal-Eiras A, Croce MV. Invasive breast cancer in Argentine women: association between risk and prognostic factors with antigens of a peptidic and carbohydrate nature. Breast Cancer (Dove Med Press). 2011;3:161-173.

27. Rabassa ME, Croce MV, Pereyra A, Segal-Eiras A. MUC1 expression and anti-MUC1 serum immune response in head and neck squamous cell carcinoma (HNSCC): a multivariate analysis. BMC Cancer. 2006;6(1):253.

28. Croce MV, Rabassa ME, Pereyra A, Segal-Eiras A. Differential expression of MUC1 and carbohydrate antigens in primary and secondary head and neck squamous cell carcinoma. Head Neck. 2008;30(5):647-657.

29. Croce MV, Price M, Segal-Eiras A. Antigen heterogeneity in advanced colorectal and breast cancer. Int J Oncol. 1996;9:459-63.

30. Zwenger A, Rabassa M, Demichelis S, Grossman G, Segal-Eiras A, Croce MV. High expression of sLex associated with poor survival in Argentinian colorectal cancer patients. Int J Biol Markers. 2014;29(1):e30-e39.

31. Lo-Guidice JM, Merten MD, Lamblin G, et al. Mucins secreted by a transformed cell line derived from human tracheal gland cells. Biochem J. 1997;326(Pt 2):431-437.

32. Zhang K, Sikut R, Hansson GC. A MUC1 mucin secreted from a colon carcinoma cell line inhibits target cell lysis by natural killer cells. Cell Immunol. 1997;176(2):158-165.

33. Lindén SK, Sheng YH, Every AL, et al. MUC1 limits Helicobacter pylori infection both by steric hindrance and by acting as a releasable decoy. PLoS Pathog. 2009;5(10):e1000617.

34. Ceriani RL, Chan CM, Baratta FS, Ozzello L, DeRosa CM, Habif DV. Levels of expression of breast epithelial mucin detected by monoclonal antibody BrE-3 in breast-cancer prognosis. Int J Cancer. 1992;51(3):343-354.

35. Rahn JJ, Dabbagh L, Pasdar M, Hugh JC. The importance of MUC1 cellular localization in patients with breast carcinoma: an immunohistologic study of 71 patients and review of the literature. Cancer. 2001;91(11):1973-1982.

36. Patton S, Gendler SJ, Spicer AP. The epithelial mucin, MUC1, of milk, mammary gland and other tissues. [review]. Biochim Biophys Acta. 1995;1241(3):407-423.

37. Croce MV, Isla-Larrain MT, Demichelis SO, Gori JR, Price MR, Segal-Eiras A. Tissue and serum MUC1 mucin detection in breast cancer patients. Breast Cancer Res Treat. 2003;81(3):195-207.

38. Horm TM, Schroeder JA. MUC1 and metastatic cancer: expression, function and therapeutic targeting. Cell Adhes Migr. 2013;7(2):187-198.
39. Lacunza E, Baudis M, Colussi AG, Segal-Eiras A, Croce MV Abba MC. MUC1 oncogene amplification correlates with protein overexpression in invasive breast carcinoma cells. Cancer Genet Cytogenet. 2010;201(2):102-110.

40. Altschuler $\mathrm{Y}$, Kinlough $\mathrm{CL}$, Poland PA, et al. Clathrin-mediated endocytosis of MUC1 is modulated by its glycosylation state. Mol Biol Cell. 2000;11(3):819-831.

41. Merlin J, Stechly L, de Beaucé S, et al. Galectin-3 regulates MUC1 and EGFR cellular distribution and EGFR downstream pathways in pancreatic cancer cells. Oncogene. 2011;30(22): 2514-2525.

42. Kumar $\mathrm{P}$, Lindberg $\mathrm{L}$, Thirkill TL, et al. The MUC1 extracellular domain subunit is found in nuclear speckles and associates with spliceosomes. PLoS One. 2012;7(8):e42712.

43. Croce MV, Isla-Larrain M, Tur R, Rabassa ME, Segal-Eiras A. Antigenic differences between metastatic cells in bone marrow and primary tumours and the anti-MUC1 humoral immune response induced in breast cancer patients. Clin Exp Metastasis. 2004;21(2):139-147.

44. Demichelis SO, Alberdi CG, Servi WJ, Isla-Larrain MT, Segal-Eiras $A$, Croce MV. Comparative immunohistochemical study of MUC1 and carbohydrate antigens in breast benign disease and normal mammary gland. Appl Immunohistochem Mol Morphol. 2010;18(1):41-50.

45. McAuley JL, Linden SK, Png CW, et al. MUC1 cell surface mucin is a critical element of the mucosal barrier to infection. J Clin Invest. 2007;117(8):2313-2324.

46. Thirkill TL, Cao T, Stout M, Blankenship TN, Barakat A, Douglas GC.. MUC1 is involved in trophoblast transendothelial migration. Biochim Biophys Acta. 2007;1773(6):1007-1014.

47. Francis LW, Lewis PD, Gonzalez D, et al. Progesterone induces nano-scale molecular modifications on endometrial epithelial cell surfaces. Biol Cell. 2009;101(8):481-493.

48. Cascio S, Zhang L, Finn OJ. MUC1 protein expression in tumor cells regulates transcription of proinflammatory cytokines by forming a complex with nuclear factor- $\mathrm{kB}$ p 65 and binding to cytokine promoters: importance of extracellular domain. J Biol Chem. 2011;286:42248-42256.

49. Kosugi S, Hasebe M, Tomita M, Yanagawa H. Systematic identification of cell cycle-dependent yeast nucleocytoplasmic shuttling proteins by prediction of composite motifs. Proc Natl Acad Sci USA. 2009;106(25):10171-10176.

50. Kosugi S, Hasebe M, Matsumura N, et al. Six classes of nuclear localization signals specific to different binding grooves of importin $\alpha$. J Biol Chem. 2009;284(1):478-485.

51. Ng W, Loh AXW, Teixeira AS, Pereira SP, Swallow DM. Genetic regulation of MUC1 alternative splicing in human tissues. $\mathrm{Br} J$ Cancer. 2008;99(6):978-985.

52. Obermair A, Schmid BC, Stimpfl M, et al. Novel MUC1 splice variants are expressed in cervical carcinoma. Gynecol Oncol. 2001;83(2):343-347.

53. Weiss M, Baruch A, Keydar I, Wreschner DH. Preoperative diagnosis of thyroid papillary carcinoma by reverse transcriptase polymerase chain reaction of the MUC1 gene. Int J Cancer. 1996;66(1):55-59.

54. Schmid BC, Buluwela L, Liu Q, et al. Expression of $\mathrm{MUCl}$ splice variants correlates with invasive growth of breast cancer cell lines. Breast Cancer Res Treat. 2002;76(3):211-219.

55. Schmid BC, Rudas M, Fabjani G, et al. Evaluation of MUC1 splice variants as prognostic markers in patients with ductal carcinoma in situ of the breast. Oncol Rep. 2003;10(6): 1981-1985. 